\title{
An Algorithm for Calculating Collision Probability of Spacecraft and Short-term Debris Cloud Based on Binomial Distribution
}

\author{
Yue Qi ${ }^{1, a,{ }^{*}}$, Jiazhe Lai ${ }^{2, b}$, Yiyong Li ${ }^{2, c}$ and Qichen Tian ${ }^{1, d}$ \\ 1Department of Graduate Management, Equipment Academy of PLA, Beijing 101416, China \\ ${ }^{2}$ Department of Space Command, Equipment Academy of PLA, Beijing 101416, China \\ a974791993@qq.com, b15201679976@163.com, c15605192191@163.com, \\ d15651756195@163.com
}

Keywords: spacecraft; space collision; debris cloud; collision probability.

\begin{abstract}
This paper introduces the calculation process of collision probability between spacecraft and short-term debris cloud. On the base of the time integral method about collision probability calculation, the time required for the spacecraft to travel through the debris cloud by the way of "risk radius"; On the base of "quasi mean probability" and the form of binomial distribution, reestablished the mathematical expression for calculating the spacecraft and space debris collision probability, which is more consistent with the actual situation. Finally, by sample analysis, the rationality of the analytical method is validated.
\end{abstract}

\section{Introduction}

As the increasing of space activities in the world, there are more and more spacecraft in orbit. As of February 2017, in earth orbit, orbit target of up to 17500 per SSN catalogue and the on orbit spacecraft is only about 4000 (including the failure of the spacecraft), the rest could be classified as of the space debris [1]. As the data says, the number of space debris is very huge, no doubt that such a large team in space running aimlessly to the safety of the spacecraft effectively caused a great threat. Therefore, the analysis of the threat posed by space debris or short-term debris clouds to the spacecraft has to be considered in the flight process.

Many foreign countries have established the corresponding environmental model. However, these models give the average flux for the long-term evolution of debris, and can not be applied to the calculation of the collision probability between spacecraft and short-term debris clouds. Ting Wang systematically described the calculation method of short-term debris cloud and spacecraft collision probability; Yiyong Li established the method which could rapidly screen out the dangerous spacecraft, and put forward the "quasi mean probability" method. This paper will base on these works, and calculate the time of spacecraft through short-term debris cloud by the way of "risk zone radius"; On the basic of binomial distribution and "quasi mean probability", the paper will calculate the collision probability between spacecraft and debris cloud to assess the hazards of debris cloud.

\section{The process of collision probability calculation}

The collision probability involves 5 kinds of collision probability [2]. Their names and relationships are shown in Fig.1. First, the probability density function of the debris cloud at any time is derived according to the probability density function of the velocity of the collision breakup model. The second step is to calculate the collision probability between spacecraft and debris cloud. The third step is to calculate the collision probability after considering the orbit error. 


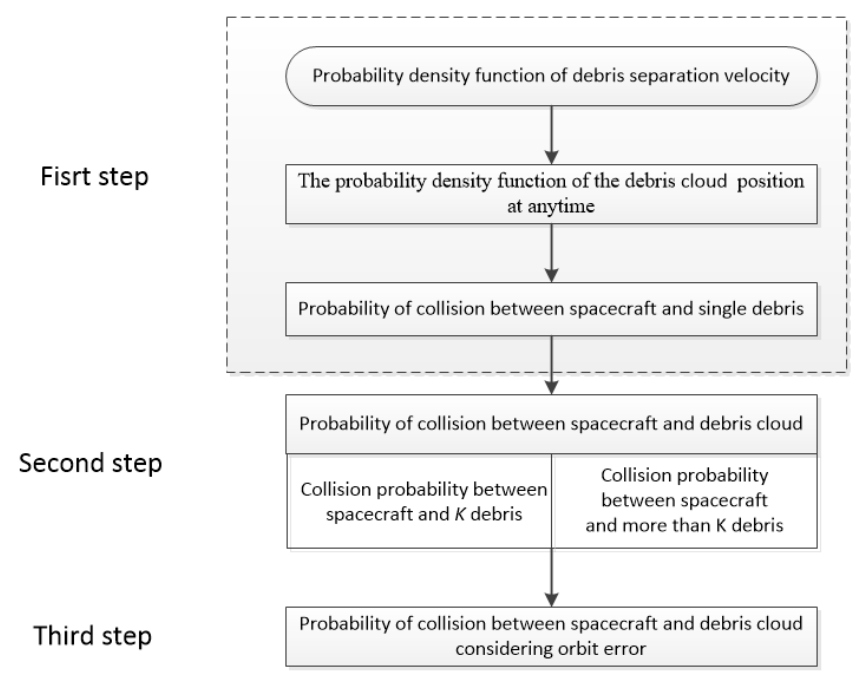

Figure 1. The relation of every collision probability and calculation process

\section{Collision Probability Calculation Method}

\subsection{Collision Probability Calculation Method for Spacecraft and Single Debris}

The methods for solving the problem include integral method and Monte Carlo method.

Monte Carlo method is that when a problem is a probability of random events occur, or the expected value of a random variable, through some method of "experiment", in this event the probability of the frequency estimation of a random event.

The integral method is divided into time integral method and spatial integral method. The idea is based on the $\mathrm{C}-\mathrm{W}$ equation, the spacecraft collision breakup model of velocity distribution and the probability density function of spacecraft relative motion in the position probability density function of debris cloud at any time. It is a description of the specific distribution of debris in space at a moment

According to the probability density function of the position, $p_{0}$ which is the probability of collision between each fragment and the spacecraft in the time of the spacecraft passing through the debris cloud can be obtained.

$$
p_{0}=\int g(t) V_{r}(t) S d t
$$

In the formula, $g(t)$ is the probability density function of debris position; $V_{r}(t)$ is the relative velocity of spacecraft and debris; $S$ is the cross-sectional area of the spacecraft.

Due to the wide applicability of the time integration method, and in the process of solving the space integral method is easy to solve, so this paper mainly uses the probability calculation of the time integration method.

\subsection{Probability Density Function Algorithm for Debris Delta-velocity}

Spacecraft collision breakup model is the basis and key to solve the problem of space high speed collision disintegration. According to the SCBM model, the size $L_{c n}$ of the nth fragment can be obtained by the size distribution formula, which can be obtained from the size of the order. [3]

$$
L_{c n}=(A / n)^{\frac{1}{2.28 B}}\left(\frac{m_{t o t}}{5.9219}\right)^{\frac{1}{2.28}}, n=1,2,3 \cdots
$$

In the formula, $A$ is the scale factor; $B$ is the power coefficient; $m_{t o t}$ is the total disintegration mass.

According to the model of debris area-mass ratio, than get $D_{A / M}\left(\lambda_{c n}, \chi\right)$ the description of debris area-mass ratio by probability density function; according to the probability density function of the model, in order to get the delta-velocity of the probability density function of the debris $D(\delta)$

$$
D(\delta)=\int D_{A / M}\left(\lambda_{c n}, \chi\right) D_{\Delta v}(\chi, \delta) d \chi
$$


In the formula, $\lambda_{c n}=\lg \left(L_{c n}\right)$ is the logarithm of the characteristic, $\mathrm{m} ; \chi=\lg (A / M)$ is the $\operatorname{logarithm}$ of area-mass ratio $A / M, \mathrm{~m}^{2} / \mathrm{kg} ; \delta=\lg (\Delta v)$ is the logarithm of delta-velocity, $\mathrm{m} / \mathrm{s}$. Based on the formula (4), the probability density function of the delta-velocity is derived under the assumption that the velocity direction of the delta-velocity, described is [3]

$$
D(\Delta v)=\frac{1}{\Delta v \cdot \ln 10} \int D_{A / M}\left(\lambda_{c n}, \chi\right) D_{\Delta v}(\chi, \delta) d \chi
$$

The delta-velocity is assumed to be uniformly distributed, so the probability density function can be expressed as

$$
D_{d i r}(\Delta \vec{v})=\frac{1}{4 \pi \cdot \Delta v^{2}}
$$

The probability density function of the delta-velocity $f(\Delta \vec{v})$ is obtained

$$
f(\Delta \vec{v})=D_{\text {dir }}(\Delta \vec{v}) D(\Delta v)
$$

The probability density function of the delta-velocity depends on the characteristic size of each fragment, that is to say, the fragmentation probability density function can be obtained from the different characteristic sizes.

\subsection{Method for Calculating Probability Density Function of Debris Position}

A method for calculating the probability density function of fragment position in [8] is proposed: Supposing that the spacecraft separated a piece of debris after being hit and after time $t$, the displacement vector of the debris is $\boldsymbol{r}$, velocity vector is $\boldsymbol{v}$. In the position space and velocity space, we can find a micro element of the position space $d \boldsymbol{r}$ and a velocity vector $d \boldsymbol{v}$

$$
g(\boldsymbol{r}) d \boldsymbol{r}=f(\boldsymbol{v}) d \boldsymbol{v}
$$

In the formula, $f_{v}()$ is the probability density function of delta-velocity; $f_{\rho}()$ is the probability density function of position. The relation between the position vector element and the velocity vector element can be expressed by the following formula

$$
d r=|J| d v
$$

In the formula, $J$ is the Jacoby conversional matrix between position space and velocity space.

The probability density function of the fragment can be obtained by (9) and (4)

$$
g(\boldsymbol{r})=\frac{1}{|\boldsymbol{J}|} f(\boldsymbol{v})
$$

\subsection{Time Integration Method for Single Fragment Collision Probability}

When the debris and spacecraft relative motion to meet the conditions of application of the $\mathrm{C}-\mathrm{W}$ equation, namely the debris cloud center for the circular orbit or eccentricity is very small; the debris delta-velocity of debris cloud is small; the evolution time of no more than half an orbital period. We can establish the orbital coordinate system ( $\mathrm{Z}$ axis downward toward the earth, $\mathrm{X}$ axis in the orbital plane perpendicular to the $\mathrm{Z}$ axis $\mathrm{Y}$ axis perpendicular to the plane of the orbit forward, right) in the center of debris cloud as the origin, the establishment of fragments with respect to kinematic equations debris cloud center [4].

$$
\boldsymbol{r}=\boldsymbol{B}(t) \boldsymbol{v}
$$

We put the formula (12) into the formula (11), then we can obtained $g(\boldsymbol{r})$

$$
g(\boldsymbol{r})=\frac{1}{\|\boldsymbol{B}(t)\|} f\left(\boldsymbol{B}(t)^{-1} \boldsymbol{r}\right)
$$

At the same time, using the $\mathrm{C}-\mathrm{W}$ equation to describe the relative motion of the spacecraft relative to the center of the debris cloud in the orbital coordinate system of the debris cloud, the form is as follows[5]

$$
\left[\begin{array}{c}
\boldsymbol{R}_{e}(t) \\
\boldsymbol{V}_{e}(t)
\end{array}\right]=\left[\begin{array}{ll}
\boldsymbol{A}(\tau) & \boldsymbol{B}(\tau) \\
\boldsymbol{C}(\tau) & \boldsymbol{D}(\tau)
\end{array}\right]\left[\begin{array}{l}
\boldsymbol{R}_{\boldsymbol{e} 0} \\
\boldsymbol{V}_{\boldsymbol{e} \boldsymbol{0}}
\end{array}\right]
$$


In the formula, $\boldsymbol{R}_{\boldsymbol{e} \boldsymbol{\theta}}=\boldsymbol{R}_{\boldsymbol{e}}\left(t_{0}\right)$ is the initial displacement of spacecraft into debris cloud; $\boldsymbol{V}_{\boldsymbol{e} \boldsymbol{\theta}}=V_{e}\left(t_{0}\right)$ is the initial velocity of spacecraft into debris cloud; $\boldsymbol{R}_{e}(t)\left[r_{e x}, r_{e y}, r_{e z}\right]^{T}$ is the displacement of spacecraft after $\tau=t-t_{0}$ seconds; $V_{e}(t)\left[v_{e x}, v_{e y}, v_{e z}\right]^{T}$ is the velocity of spacecraft into debris cloud after $\tau=t-t_{0}$ seconds. $\boldsymbol{A}(\tau), \boldsymbol{B}(\tau), \boldsymbol{C}(\tau), \boldsymbol{D}(\tau)$ is based on the $\mathrm{C}-\mathrm{W}$ equation, the relative motion state transition matrix is established in document[5].

We put the formula (12) into the formula (14), then we can obtained $g(t)$

$$
g(t)=\frac{1}{\|\boldsymbol{B}(t)\|} f\left(\boldsymbol{B}(t)^{-1} R_{e}(t)\right)
$$

$\boldsymbol{V}_{r}(t)$ in formula (1) is

$$
\boldsymbol{V}_{r}(t)=\boldsymbol{V}_{d}(t)-\boldsymbol{V}_{e}(t)
$$

So

$$
p_{0}=S \int_{t_{0}}^{T+t_{0}} \frac{f\left(B(t)^{-1} R_{e}(t)\right)\left|D(t) B(t)^{-1} R_{e}(t)-V_{e}(t)\right|}{\|B(t)\|} d t
$$

Among them, for the spacecraft through the debris cloud time, according to the definition of the radius of the risk region in the document [4] and the relative velocity between the debris cloud center and the spacecraft. Radius of risk zone $R$

$$
R=\sqrt{\frac{N S}{4 \pi p_{d}}}
$$

In the formula, $N$ is the number of fragments produced by the collision of spacecraft; $S$ is the crosssectional area; $p_{d}$ is the threshold value of the probability of collision. So by the time mathematical expression of the debris cloud is

$$
T=2 \sqrt{\frac{N S}{4 \pi p_{d} V_{e}\left(t_{0}\right)^{2}}}
$$

\subsection{Collision Probability Between Spacecraft and Debris Cloud}

In the document [2], the collision problem between spacecraft and debris cloud is simplified into a Bernoulli test, and the binomial distribution of parameters is considered as $N$ and $p_{0}$. However, different sizes of debris have different separation velocity density functions, so the collision probability between spacecraft and debris is not a fixed value, the direct use of the binomial distribution and Poisson distribution will cause greater error.

If you need to further the probability of collision between the spacecraft and debris, you need to use the method of free combination, because the total number of samples is very large, so that the amount of calculation surge. The method of "quasi mean probability" is proposed in document [4]

$$
\bar{p}=\frac{\bar{p}_{0}+\bar{p}_{N}}{2}
$$

In the formula, $\bar{p}_{0}=1-\left[\prod_{n=1}^{N}\left(1-p_{n}\right)\right]^{\frac{1}{N}}, \bar{p}_{N}=\left(\prod_{n=1}^{N} p_{n}\right)^{\frac{1}{N}}$ express the actual average probability of $k=0$ and $k=N$.

In this paper, the probability calculation method based on the " binomial distribution " is put forward, and the mathematical expressions are as follows.

$$
p_{N, k}(X=k)=C_{N}^{k} \frac{\prod_{i=1}^{N}\left(1-p_{i}\right)}{(1-\bar{p})^{k}}(\bar{p})^{k}
$$

The probability of collision between the spacecraft and more than $k$ debris is 


$$
p_{k}\{X \geq k\}=1-\sum_{i=0}^{k-1} p_{N, i},(k=1,2,3, \cdots)
$$

\section{Case Calculation}

Case conditions: The orbital elements of the spacecraft and the debris cloud spacecraft are shown in table 1 . The debris cloud mass is $990 \mathrm{~kg}$, the impact mass is $10 \mathrm{~kg}$, the impact velocity is $10 \mathrm{~km} / \mathrm{s}$. The cross sectional area of the spacecraft is $10 \mathrm{~m}^{2}$.

Table 1. Original Orbital Elements

\begin{tabular}{|c|c|c|c|c|c|c|}
\hline Parameter & $\begin{array}{c}\text { Semi-major } \\
\text { axis } \\
a / \mathrm{km}\end{array}$ & Eccentricity $e$ & $\begin{array}{c}\text { Orbital } \\
\text { inclination } \\
i /\left(^{\circ}\right)\end{array}$ & $\begin{array}{c}\text { Ascension } \\
\text { of } \\
\text { ascending } \\
\text { node } \\
\Omega /\left(^{\circ}\right)\end{array}$ & $\begin{array}{c}\text { Perigee } \\
\text { argument } \\
\omega /\left(^{\circ}\right)\end{array}$ & $\begin{array}{c}\text { True } \\
\text { perigee } \\
\text { angle } \\
f /\left(^{\circ}\right.\end{array}$ \\
\hline $\begin{array}{c}\text { Debris cloud } \\
\text { Center }\end{array}$ & 6875.137 & 0 & 97.00 & 0.003 & 0 & 347.333 \\
\hline $\begin{array}{c}\text { Target } \\
\text { spacecraft }\end{array}$ & 6878.137 & 0 & 80.00 & 359.998 & 0 & 347.334 \\
\hline
\end{tabular}

According to the model of SCBM impact disintegration, the collision produced a total of 40974 above $1 \mathrm{~cm}$ fragments. The collision probability with 1 pieces at least, and probability of collision with 1 fragments, probability of collision with 2 fragments and probability of collision with 3 fragments..... The results are shown in Table 2.

Table 2. The Results of Collision Probability between Spacecraft and Short-term Debris Cloud

\begin{tabular}{|c|c|c|c|c|c|l|}
\hline \multirow{2}{*}{ Project } & $\begin{array}{c}\text { Quasi mean } \\
\text { probability }\end{array}$ & $\begin{array}{c}\text { Collision } \\
\text { probability } \\
p_{1}\{X \geq 1\}\end{array}$ & $\begin{array}{c}\text { Collision } \\
\text { probability } \\
\text { with } 2 \\
\text { fragments } \\
p_{N, 1}(X=1)\end{array}$ & $\begin{array}{c}\text { Collision probability } \\
\text { with 2 fragments } \\
p_{N, 2}(X=2)\end{array}$ & $\begin{array}{c}\text { Collision probability } \\
\text { with } 3 \text { fragments } \\
p_{N, 3}(X=3)\end{array}$ & $\ldots$ \\
\hline Results & $3.0720 \times 10^{-7}$ & 0.0129 & 0.0127 & $8.0218 \times 10^{-5}$ & $3.3655 \times 10^{-7}$ & $\ldots$ \\
\hline
\end{tabular}

\section{Conclusion}

This paper introduces the calculation method of spacecraft and short-term debris cloud collision probability, based on using the previous provisions, the spacecraft through the debris cloud; provide a method to calculate the collision probability of quasi average probability based on the distribution of two, compared with the pure Poisson distribution calculation is more practical and the previous algorithm, the original data is fully utilized, instead of using the average value of the collision probability in the sample instead of every debris collision probability. Finally, a simulation example is given to analyze and verify the results.

\section{Acknowledgments}

Here, first of all, I would like to thank my mentor Li Yi-yong for my guidance, and other people who helped me in the writing of this thesis.

The main research direction of this paper is the evolution of debris cloud. On the basis of previous studies, this paper proposes a new method to calculate the collision probability between the spacecraft and the debris cloud. 


\section{References}

[1] Orbital Debris Quarterly News. Volume 21, Issue 1 February 2017.

[2] Wang TIng, Dong Yun-feng, Algorithms of Collision Probability between Spacecraft and Short Term Debris Clouds. Chinese space science and technology, 2006, 26(2):17-23.

[3] Shen Huai-rong, Modeling and Analysis of Spacecraft Impact (The Science Publishing Company, Beijing, 2014).

[4] Li Yi-yong, Shen Huai-rong, Li Zhi.Assessing the Short-Term Hazard of Spacecraft Collision Breakup Debris. Journal of Astronautics, 2010, 31(4):1231-1236.

[5] Sheng Zhou. Probability theory and mathematical statistics (Higher Education Press, Beijing, 2001).

[6] Patera R P. Method for Calculating Collision Probability between a Satellite and a Space Tether [J]. Journal of Guidance Control \& Dynamics, 2002, 25(5):págs. 940-945.

[7] Housen K R. The short-term evolution of orbital debris clouds [J]. Journal of the Astronautical Sciences, 1992, 40(2):203-213. 